\title{
Differential gene expression and cytokine production from neutrophils in asthma phenotypes
}

\author{
K.J. Baines*,\# , J.L. Simpson*,\# , N.A. Bowdenๆ, R.J. Scott ${ }^{\top}$ and P.G. Gibson*,\#
}

ABSTRACT: Asthma is characterised into eosinophilic and non-eosinophilic phenotypes based on inflammatory cell patterns in airway secretions. Neutrophils are important in innate immunity, and are increased in the airways in non-eosinophilic asthma. The present study investigated the activity of neutrophils in asthma phenotypes.

Participants with eosinophilic $(n=8)$ and non-eosinophilic asthma $(n=9)$ and healthy controls $(n=11)$ underwent sputum induction and blood collection. Neutrophils were isolated and cultured with or without lipopolysaccharide. Cytokines were measured by ELISA, and gene expression was analysed using a gene expression microarray and quantitative PCR.

In non-eosinophilic asthma, blood neutrophils released significantly higher levels of interleukin-8 at rest. Cytokine gene expression and sputum neutrophil protein production did not differ between asthma subtypes. Microarrays demonstrated closely related expression profiles from participants with non-eosinophilic asthma that were significantly distinct from those in eosinophilic asthma. A total of $\mathbf{3 1 7}$ genes were significantly altered in resting neutrophils from participants with non-eosinophilic asthma versus eosinophilic asthma, including genes related to cell motility and regulation of apoptosis.

Non-eosinophilic and eosinophilic asthma are associated with specific gene expression profiles, providing further evidence that these phenotypes of asthma involve different molecular mechanisms of disease pathogenesis at the systemic level. The mechanisms of non-eosinophilic asthma may involve enhancement of blood neutrophil chemotaxis and survival.

KEYWORDS: Airway inflammation, asthma phenotypes, gene expression, innate immunity, interleukin-8, neutrophils

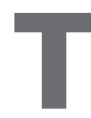
he inflammatory response in asthma is heterogeneous, involving a well-characterised eosinophilic pathway that is triggered by the inhalation of allergens, and which involves activation of type-2 T-helper lymphocytes and interleukin (IL)-5 production. Non-eosinophilic asthma represents an alternative asthma phenotype in which patients exhibit asthma symptoms and heightened airway responsiveness in the absence of significant eosinophilia [1-4]. The mechanisms underlying non-eosinophilic inflammation in asthma are unclear; however, neutrophils may be important since studies of non-eosinophilic asthma find increased numbers of neutrophils and elevated levels of the neutrophil chemoattractant IL-8 in the airways [5]. Furthermore, neutrophilic asthma is associated with innate immune activation, specifically increases in the expression of the
Toll-like receptors (TLRs) TLR2, TLR4 and CD14, as well as the pro-inflammatory cytokines IL- 8 and IL$1 \beta$, in airway samples [6]. The levels of these innate immune mediators measured in the sputum correlate with the number of neutrophils in the airways, implicating a role for neutrophils in the local production of these mediators.

Neutrophils have long been considered phagocytes whose main purpose is to engulf and degrade microorganisms. However, recent microarray studies have provided substantial evidence that neutrophils are capable of extensive gene expression changes that are important in the regulation of many neutrophil functions, as well as modulation of the immune response. A wide range of genes are expressed in unstimulated neutrophils, and this gene profile is dramatically changed in response to bacterial exposure [7], transmigration to the

\section{AFFILIATIONS}

*Priority Research Centre for Asthma and Respiratory Diseases, The University of Newcastle,

- Priority Research Center for Information Based Medicine, Hunter Medical Research Institute, The University of Newcastle, Callaghan, and

\#Dept of Respiratory and Sleep

Medicine, Hunter Medical Research Institute, John Hunter Hospital, New Lambton, Australia.

CORRESPONDENCE

K.J. Baines

Level 3, Hunter Medical Research Institute

John Hunter Hospital

Locked Bag 1

Hunter Region Mail Centre

Newcastle

NSW 2310

Australia

E-mail: katherine.baines@

newcastle.edu.au

Received:

Feb 172009

Accepted after revision:

Sept 092009

First published online:

Sept 242009 
airways [8] and neutrophil-mediated diseases [9]. Marked changes in neutrophil gene expression occur following experimental exposure to soluble lipopolysaccharide (LPS) $[8,10]$ and whole bacteria [11]. LPS, a potent stimulus of innate immune responses, leads to alterations in gene expression that include genes that encode cytokines and receptors, genes involved in host defence, apoptosis-related genes, genes encoding transcription factors and chromatin-remodelling genes [11].

Although neutrophils are present in increased numbers in noneosinophilic asthma, the precise mechanisms of their recruitment and accumulation remain largely unknown. Wholegenome gene expression analysis has not been widely used to investigate the molecular mechanisms underlying asthma, but could provide useful information relating to the heterogeneity of disease. The present study investigated the activation of circulating and sputum neutrophils in non-eosinophilic asthma, including the production of innate immune mediators, specifically the pro-inflammatory cytokines IL-8, IL-1 $\beta$, tumour necrosis factor (TNF)- $\alpha$ and oncostatin M (OSM), the expression of TLR2 and TLR4, and whole-genome gene expression using microarrays. We hypothesised that neutrophils would show increased activation in non-eosinophilic asthma compared to eosinophilic asthma, demonstrated by increased protein release and expression of important innate immune mediator genes.

\section{MATERIALS AND METHODS Participants}

Nonsmoking adults with stable asthma $(n=17)$ were defined using the American Thoracic Society criteria, had a doctor's diagnosis of symptomatic asthma and demonstrated evidence of airways hyperresponsiveness to hypertonic saline. Healthy controls $(n=11)$ exhibited no respiratory symptoms, with normal spirometric results and airways hyperresponsiveness. Participants were excluded if they had had a course of oral corticosteroids, antibiotics or a respiratory infection within 4 weeks prior to the visit. Participants were recruited through the Respiratory Ambulatory Care Service at the John Hunter Hospital (New Lambton, Australia) or by advertisement (healthy controls) and underwent clinical assessment, an allergy skin-prick test, spirometry, sputum induction and blood collection. All participants gave informed consent prior to their inclusion in the study and the Hunter Area Health Service and The University of Newcastle (Callaghan, Australia) Research Ethics Committees approved this study.

\section{Sputum induction and analysis}

Spirometry (KoKo; PD Instrumentation, Louisville, CO, USA) and sputum induction with hypertonic saline $(4.5 \%)$ were performed as previously described [12]. A fixed sputum induction time of $15 \mathrm{~min}$ was used for all participants. Selected sputum was dispersed using dithiothreitol. The suspension was filtered, and total leukocyte count and cell viability were determined. Cytospins were prepared and stained (May-Grünwald Giemsa), and a differential cell count obtained from 400 nonsquamous cells.

\section{Asthma subtype classification}

Based on previous studies [2], participants with a sputum eosinophil count of $\geqslant 1 \%$ alone were classified as having eosinophilic asthma, and participants with sputum eosinophil counts of $<1 \%$ were classified as having non-eosinophilic asthma. Those participants with increased neutrophils ( $>63 \%)$ and eosinophils $(>1 \%)$ were classified as having non-eosinophilic asthma [2].

\section{Neutrophil isolation and culture}

Peripheral blood neutrophils were isolated from $50 \mathrm{~mL}$ whole blood using a Percoll density gradient and magnetic cell separation using CD16 microbeads (Miltenyi Biotec, Gladbach, Germany). CD16-positive cells were isolated from the remainder of the sputum sample using magnetic cell separation. Highly pure blood neutrophils (median 100\% (interquartile range $96-100 \%)$ ) and the neutrophil-enriched cell fraction (59\% (30-78\%) neutrophils; 35\% (22-57\%) macrophages) were cultured with or without LPS $\left(100 \mathrm{ng} \cdot \mathrm{mL}^{-1}\right)$ for $24 \mathrm{~h}$. Further details are provided in the supplementary material.

\section{Detection of mediators}

Cytokine production was assessed from isolated airway and peripheral blood neutrophils after $24 \mathrm{~h}$ of culture. The concentrations of IL-8, IL-1 $\beta$, TNF- $\alpha$ and OSM were determined by ELISA (R\&D Systems, Minneapolis, MN, USA). The standard curves for these assays ranged $31.3-2,000 \mathrm{pg} \cdot \mathrm{mL}^{-1}$ for IL-8, TNF- $\alpha$ and OSM, and $7.8-250 \mathrm{pg} \cdot \mathrm{mL}^{-1}$ for IL-1 $\beta$. Target gene expression was analysed using real-time PCR. RNA was prepared and reverse transcribed to cDNA as described previously [13]. PCR probes were purchased in kit form (Applied Biosystems, Foster City, CA, USA). PCR primers and probes were combined with the reference gene eukaryotic $18 \mathrm{~S}$ ribosomal RNA in duplex real-time PCRs as previously described (7500 Real-Time PCR System; Applied Biosystems) [13]. The amount of target present was calculated relative to the $18 \mathrm{~S}$ housekeeping gene and an internal calibrator $\left(2^{-\Delta \Delta \mathrm{Ct}}\right.$ where $\Delta$ is change and $\mathrm{Ct}$ is the cycle threshold).

\section{Gene expression profiling}

Selected blood neutrophil samples were processed for gene expression analysis, including those from four participants with non-eosinophilic asthma who had sputum neutrophil counts of $>63 \%$ and five with eosinophilic asthma who had sputum eosinophil counts of $>2.5 \%$. RNA was extracted using the RNeasy Mini Kit (Qiagen, Hilden, Germany) and quantified using the Quant-iT RiboGreen RNA Quantitation Assay Kit (Molecular Probes, Inc, Invitrogen, Eugene, OR, USA). Fluorescence was measured at wavelengths of $485 \mathrm{~nm}$ for excitation and $520 \mathrm{~nm}$ for emission (FLUOstar Optima; BMG LABTECH, Mornington, Australia). A total of $500 \mathrm{ng}$ RNA was reverse transcribed into $\mathrm{CRNA}$ and labelled with biotin-uridine triphosphate using the Illumina TotalPrep RNA Amplification Kit (Ambion, Austin, TX, USA). A total of 850 ng cRNA was hybridised to Illumina Sentrix HumanRef-8 v1.1 Expression BeadChips (Illumina, San Diego, CA, USA) using standard protocols. Each BeadChip measured the expression of 24,354 genes and was scanned using the Illumina Bead Station and captured using BeadScan 3.5.11 (Illumina).

\section{Statistical analysis}

Data were analysed using Stata 9 (Stata Corporation, College Station, TX, USA). All data, unless otherwise stated, are nonparametric and reported as median (interquartile range). In the case of age, percentage predicted forced expiratory volume in 
$1 \mathrm{~s}$ (FEV1) and FEV1/forced vital capacity, data are reported as mean $\pm \mathrm{SD}$, and significant differences were determined using either the two-sample unpaired t-test or multiple-sample ANOVA. For all other data, significant differences $(p<0.05)$ were detected using the two-sample Wilcoxon rank-sum test or the multiple-sample Kruskal-Wallis test. For categorical data (sex and atopy) Fisher's exact test was applied. Associations between data were determined using Spearman's rank correlation.

For whole-genome gene expression, data were normalised using a cubic spline in the BeadStudio 2.0 software (Illumina), exported to GeneSpring 7.3.1 software (Agilent Technologies, Santa Clara, CA, USA) and further normalised to the median. Using the Wilcoxon-Mann-Whitney test, three comparisons were carried out between: 1) resting and LPS-stimulated neutrophils, 2) resting neutrophils in non-eosinophilic asthma versus eosinophilic asthma, and 3) LPS-stimulated neutrophils in non-eosinophilic asthma versus eosinophilic asthma. Using standard correlation and distance in GeneSpring 7.3.1, a dendrogram was created to show relationships between samples (Experiment Tree), and a second dendrogram to show relationships between gene expression levels across the samples (Gene Tree). Genes were judged to be differentially regulated only when: 1 ) the gene was present in all samples studied, 2) the difference in expression was $>1.5$-fold, and 3) the extent of difference in expression was significant $(p<0.05$ in Wilcoxon-Mann-Whitney test).

\section{RESULTS}

\section{Clinical features and inflammatory cells}

Clinical details and total and differential inflammatory cell counts from the induced sputum samples collected are shown in table 1 . Healthy controls $(n=11)$ without respiratory disease or symptoms exhibited an FEV1 of $>80 \%$ pred. All participants with asthma were receiving inhaled corticosteroid therapy, and $88 \%$ (15) of participants were taking combination therapy with a long-acting $\beta_{2}$-agonist. Eight ( $47 \%$ ) of the 17 participants had eosinophilic asthma, and the remaining nine (53\%) noneosinophilic asthma. The asthma pattern was classified as intermittent $(n=1 ; 6 \%)$, mild $(n=5 ; 29 \%)$, moderate $(n=6 ; 35 \%)$ or severe persistent $(n=5 ; 29 \%)$. There was no significant difference between eosinophilic and non-eosinophilic asthma regarding the clinical parameters measured; however, sputum eosinophil numbers were increased in eosinophilic asthma and sputum neutrophil numbers in non-eosinophilic asthma. Whole-genome gene expression microarray experiments were performed on selected participants with eosinophilic and noneosinophilic asthma, and their clinical details were comparable (table E1 in the supplementary material).

\section{Innate immune responses of peripheral blood neutrophils}

Resting peripheral blood neutrophils from participants with non-eosinophilic asthma released significantly more IL-8 than did those from participants with eosinophilic asthma $(p=0.03)$ (fig. 1). Resting neutrophils did not release detectable levels of TNF- $\alpha$, and 93\% (26) of resting neutrophil samples had undetectable levels of IL-1 $\beta$ and OSM. There was a trend towards upregulation of IL-8 gene expression (fig. 1b) in noneosinophilic asthma compared to eosinophilic asthma; however, OSM, IL-1 $\beta$, TNF- $\alpha$, TLR2 and TLR4 gene expression did not differ significantly between asthma phenotypes (table E2 in the supplementary material).

LPS stimulation induced the release of IL- 8, IL- $1 \beta$, TNF- $\alpha$ and OSM, and increased IL- 8 , IL-1 $\beta$, TNF- $\alpha$, OSM, TLR2 and TLR4 gene expression. LPS-stimulated neutrophils isolated from

\begin{tabular}{|c|c|c|c|c|}
\hline & \multirow[t]{2}{*}{ Controls } & \multicolumn{2}{|c|}{ Asthma } & \multirow[t]{2}{*}{ p-value } \\
\hline & & Eosinophilic & Non-eosinophilic & \\
\hline Subjects $n$ & 11 & 8 & 9 & \\
\hline Age yrs & $56 \pm 18$ & $53 \pm 20$ & $65 \pm 9$ & 0.35 \\
\hline Males/females $\mathrm{n}$ & $5 / 6$ & $4 / 4$ & $4 / 5$ & 1.0 \\
\hline $\mathrm{FEV}_{1 / \mathrm{FVC}} \%$ & $77 \pm 7$ & $70 \pm 9$ & $65 \pm 10^{\circ}$ & 0.01 \\
\hline ICS dose $^{\#} \mu \mathrm{g}$ & & $750(400-1500)$ & $1000(500-2000)$ & 0.24 \\
\hline Asthma control score & & $0.7(0.3-1.4)$ & $1.0(0.9-1.1)$ & 0.74 \\
\hline TCC $10^{6}$ cells $\cdot \mathrm{mL}^{-1}$ & $3.7(2.4-5.6)$ & $5.3(2.8-8.3)$ & $10.1(4.4-17.6)$ & 0.13 \\
\hline Neutrophils \% & $30.5(14.5-37.4)$ & $31.2(16.0-41.1)$ & $58.5(24.5-72.0)$ & 0.08 \\
\hline Neutrophils $10^{4}$ cells $\cdot \mathrm{mL}^{-1}$ & $90.9(49.1-150.7)$ & $104.7(64.4-218.7)$ & $589.7(81.6-1043.3)$ & 0.06 \\
\hline Macrophages $10^{4}$ cells $\cdot \mathrm{mL}^{-1}$ & $226.0(172.8-307.4)$ & $241.5(189.1-437.3)$ & $238.1(120.6-402.7)$ & 0.82 \\
\hline
\end{tabular}

Data are presented as mean $\pm \mathrm{SD}, \mathrm{n}(\%)$ or median (interquartile range), unless otherwise specified. FEV1: forced expiratory volume in $1 \mathrm{~s}$; \% pred: \% predicted; FVC: forced vital capacity; ICS: inhaled corticosteroid; TCC: total cell count. ${ }^{\#:} 1 \mu \mathrm{g}$ beclometasone $\equiv 1 \mu \mathrm{g}$ budesonide $\equiv 0.5 \mu \mathrm{g}$ fluticasone. ${ }^{\bullet}: \mathrm{p}<0.008$ versus healthy controls; ${ }^{+}: \mathrm{p}<0.008$ versus non-eosinophilic asthma (Kruskal-Wallis nonparametric test for significance). 

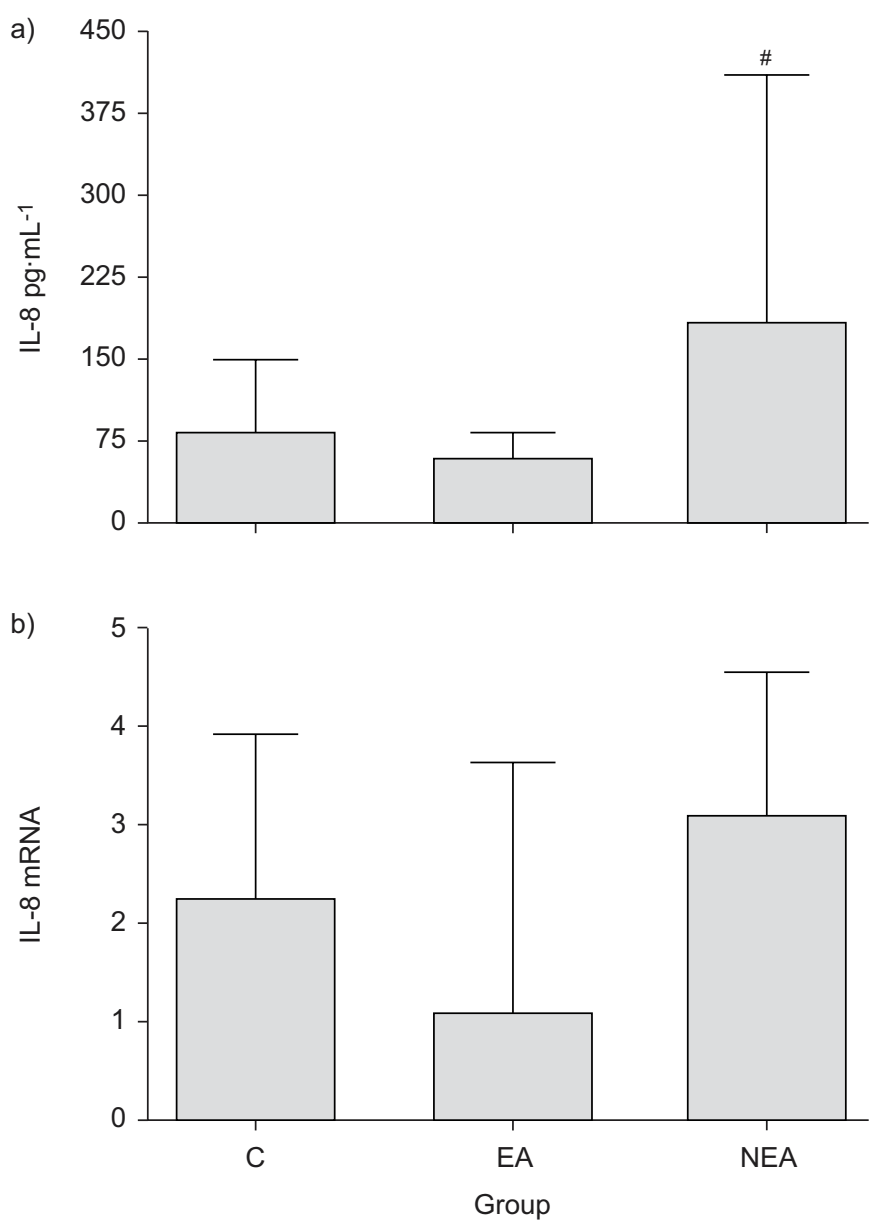

FIGURE 1. Interleukin (IL)-8 in resting blood neutrophils a) protein production and b) gene expression. Data are presented as medians; vertical bars represent the upper quartile. Gene expression is presented relative to the $18 \mathrm{~S}$ housekeeping gene. C: healthy controls $(n=11)$; EA: eosinophilic asthma $(n=8)$; NEA: noneosinophilic asthma $(n=9) .{ }^{*}: p<0.008$ versus eosinophilic asthma.

participants with eosinophilic asthma released significantly less OSM than healthy controls; however, release of IL-8, IL-1 $\beta$ and TNF- $\alpha$ was similar (fig. 2 ). IL- 8 , IL-1 $\beta$, TNF- $\alpha$, OSM, TLR2 and TLR4 gene expression did not differ significantly between asthma phenotypes in LPS-stimulated neutrophils (table E2 in the supplementary material). TLR2 and IL-1 $\beta$ gene expression was generally lower in the asthma groups than in healthy controls.

\section{Innate immune responses of sputum neutrophils}

Minimal changes in sputum neutrophils were seen between the groups. Resting sputum neutrophils from participants with non-eosinophilic asthma released significantly lower levels of TNF- $\alpha$ than did healthy controls; however, this did not differ from eosinophilic asthma (table 2). IL-8 and IL-1 $\beta$ release and IL-8, IL-1 $\beta$, TNF- $\alpha$, TLR2 and TLR4 gene expression did not differ significantly between groups in either resting or LPSstimulated sputum neutrophils, but tended, however, to be lower in both eosinophilic and non-eosinophilic asthma than in healthy controls (table 2). LPS stimulation had no effect on IL- 8 , IL-1 $\beta$, TNF- $\alpha$ and OSM release or IL- 8 , IL- $1 \beta$, TNF- $\alpha$,
OSM, TLR2 and TLR4 gene expression in sputum neutrophils. OSM was not released at detectable levels from sputum neutrophils and is, therefore, not shown.

\section{Whole-genome gene expression changes due to LPS stimulation}

Dramatic changes in gene expression were apparent between resting and LPS-stimulated circulating neutrophils isolated from participants with asthma. Using the Wilcoxon-MannWhitney test, 1,080 genes were identified with a mean expression ratio that was significantly different when comparing resting to LPS-stimulated neutrophils. As expected, the LPS-stimulated gene profile represented a pro-inflammatory state of neutrophil activation, with increases in cytokines (e.g. OSM), chemokines (e.g. IL-8, CC chemokine ligand 3-like 1 and CXC chemokine ligand 1), signalling molecules (e.g. IL-1 receptor-associated kinase (IRAK) 1 and 3), receptors (e.g. TLR2, CXC chemokine receptor 4 and CC chemokine receptor $1)$, molecules regulating apoptosis (e.g. growth arrest and DNA-damage-inducible protein (GADD) $45 \beta$, serum/glucocorticoid-regulated kinase (SGK) and CCAAT/enhancer-binding protein $\beta(\mathrm{CEBPB}))$ and components of the nuclear factor

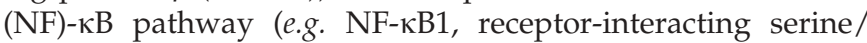
threonine-protein kinase (RIPK) 2 and TNF receptor superfamily, member 14 (TNFRSF14)). The LPS-regulated genes OSM, TLR2 and IL8 were confirmed to be upregulated via realtime PCR (table E3 in the supplementary material).

\section{Whole-genome gene expression changes due to asthma phenotype}

Resting blood neutrophils

Using the Wilcoxon-Mann-Whitney test, 317 genes from resting neutrophils were identified as exhibiting significantly different levels of expression between the asthma phenotypes. Construction of a dendrogram containing these 317 genes showed that the gene expression profiles from participants with non-eosinophilic asthma were closely related to, but significantly different from, those from the participants with eosinophilic asthma (fig. 3). In figure 3, columns represent the gene expression in resting neutrophils from each of the subjects with asthma. Downregulation is represented by green, and upregulation by red. The dendrogram at the top of the figure represents the relationship between asthma subtypes (blue branches: non-eosinophilic asthma; red branches: eosinophilic asthma), which were shown to be distinctly different. The horizontal dendrogram shows the relationship between the expression levels of each gene, i.e. genes of similar expression across the samples are grouped together.

A considerable number (54\%) of genes that were altered in resting neutrophils in non-eosinophilic asthma compared to eosinophilic asthma were also altered by LPS stimulation, suggesting that these genes play a role in neutrophil activation. Altered genes of interest with immune-related functions are shown in table 3 . These include important genes relating to neutrophil cell motility, apoptosis and the NF- $\kappa \mathrm{B}$ cascade. The expression of several genes shown in table 3 were significantly correlated with percentage predicted FEV1 (GADD45B: $r=-0.70, p=0.036$; IRAK3: $r=-0.77, p=0.016$; G-protein-coupled receptor 109B (HM74): $r=-0.72 ; \mathrm{p}=0.030$; 

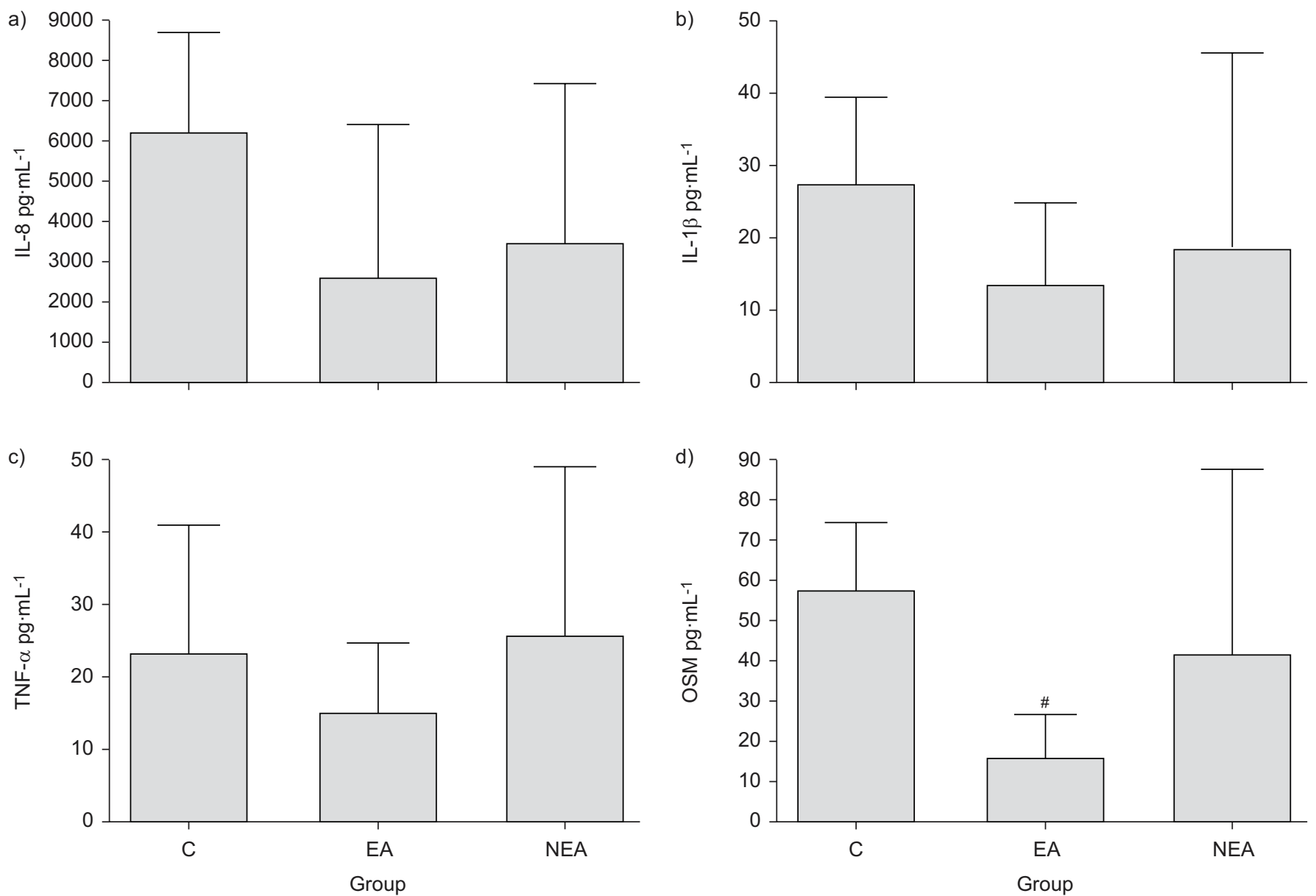

FIGURE 2. Cytokine production from lipopolysaccharide-stimulated blood neutrophils: a) interleukin (IL)-8; b) IL-1 $\beta$; c) tumour necrosis factor (TNF)- $\alpha$; and d) oncostatin M (OSM). Data are presented as medians; vertical bars represent the upper quartile. C: healthy controls $(n=11)$; EA: eosinophilic asthma $(n=8)$; NEA: non-eosinophilic asthma ( $n=9) . ~ \#: p<0.008$ versus eosinophilic asthma.

molecule possessing ankyrin repeats induced by LPS (MAIL): $\mathrm{r}=-0.78, \mathrm{p}=0.013$; protease inhibitor 3, skin derived (PI3): $r=-0.83, p=0.005(n=9))$. Further trends for correlation and between gene correlations are reported in table E4 of the supplementary material. The expression of TNFRSF14 and GADD45B were confirmed to be upregulated via real-time PCR (table E3 in the supplementary material).

\section{LPS-stimulated blood neutrophils}

Using the Wilcoxon-Mann-Whitney test, 221 genes were identified with a mean expression ratio that differed significantly between the asthma subtypes for LPS-stimulated neutrophils. Construction of a dendrogram containing these 221 genes showed that the gene expression profiles from participants with non-eosinophilic asthma were closely related to, but significantly different from, those of the participants with eosinophilic asthma. Selected genes with immune-related functions that were altered in LPS-stimulated neutrophils from participants with non-eosinophilic asthma compared to participants with eosinophilic asthma are listed in table 4. Real-time PCR results testing CC chemokine ligand 23 (CCL23) confirmed this gene to be downregulated in non-eosinophilic asthma; however, plasminogen activator, urokinase (PLAU) was unchanged between asthma subtypes (table E3 in the supplementary material).

\section{DISCUSSION}

The present study investigated activation of sputum and peripheral blood neutrophils in non-eosinophilic and eosinophilic asthma. Although there were minimal differences between groups in release of mediators from sputum cells, there were marked changes in blood neutrophils in noneosinophilic asthma. Resting blood neutrophils isolated from participants with non-eosinophilic asthma showed enhanced IL-8 release and increased IL-8 gene expression compared to participants with eosinophilic asthma, suggesting that the cells are partially activated or primed for an enhanced response. Further, whole-genome gene expression studies showed that there is a substantial degree of heterogeneity in resting neutrophils from participants with non-eosinophilic and eosinophilic asthma. In non-eosinophilic asthma, there was upregulation of genes involved in neutrophil chemotaxis, neutrophil survival and activation of the NF- $\kappa B$ cascade. This study highlights the ability of microarray technology to define inflammatory gene profiles associated with eosinophilic and non-eosinophilic asthma, and shows that there are novel and 
TABLE 2 Relative cytokine production and gene expression in resting and lipopolysaccharide (LPS)-stimulated sputum neutrophils from participants with eosinophilic and non-eosinophilic asthma and healthy controls

\begin{tabular}{|c|c|c|c|c|c|c|c|c|}
\hline & Controls & \multicolumn{2}{|c|}{ Asthma } & p-value & Controls & \multicolumn{2}{|c|}{ Asthma } & $\mathrm{p}$-value \\
\hline Subjects $n$ & 7 & 6 & 8 & & 7 & 6 & 8 & \\
\hline IL-8 mRNA & $134.4(77.2-196.7)$ & $40.5(38.1-43.7)$ & $36.9(21.8-59.1)$ & 0.06 & $77.3(42.5-102.5)$ & $51.1(48.8-71.0)$ & $62.2(45.6-113.0)$ & 0.96 \\
\hline $\mathrm{IL}-1 \beta \mathrm{pg} \cdot \mathrm{mL}^{-1}$ & $52.5(6.6-95.7)$ & $3.3(3.1-7.9)$ & $8.3(5.0-18.9)$ & 0.07 & $31.2(6.5-66.6)$ & $5.2(3.9-27.6)$ & $9.7(6.3-21.8)$ & 0.28 \\
\hline IL-1 $\beta$ mRNA & $13.5(6.8-26.5)$ & $1.7(0.8-1.9)$ & $2.8(1.0-3.9)$ & 0.07 & $7.6(2.0-13.2)$ & $2.2(1.6-4.3)$ & $4.8(2.6-5.3)$ & 0.38 \\
\hline TNF- $\alpha \mathrm{pg} \cdot \mathrm{mL}^{-1}$ & $\begin{array}{c}371.7 \\
(116.1-599.0)\end{array}$ & $\begin{array}{c}28.9 \\
(7.1-74.0)\end{array}$ & $\begin{array}{c}15.0 \\
(0.0-110.8)^{\#}\end{array}$ & 0.02 & $\begin{array}{c}257.1 \\
(117.5-550.6)\end{array}$ & $\begin{array}{c}15.0 \\
(12.4-148.9)\end{array}$ & $\begin{array}{c}31.5 \\
(1.7-124.3)\end{array}$ & 0.07 \\
\hline TNF- $\alpha$ mRNA & $4.7(1.6-5.4)$ & $0.98(0.8-1.5)$ & $1.6(0.3-3.6)$ & 0.09 & $3.2(1.9-5.0)$ & $2.0(1.3-2.4)$ & $1.1(0.4-3.5)$ & 0.46 \\
\hline
\end{tabular}

Data are presented as median (interquartile range), unless otherwise indicated. IL: interleukin; TNF: tumour necrosis factor; TLR: Toll-like receptor. ${ }^{\#}: \mathrm{p}<0.008$ versus healthy controls (Kruskal-Wallis nonparametric test for significance).

distinct gene expression profiles that relate to asthma inflammatory phenotype.

Sputum neutrophil cytokine gene expression and protein production did not differ by asthma phenotype. However, airway neutrophils generally showed lower levels of cytokine release in both eosinophilic and non-eosinophilic asthma compared to healthy controls. This only reached significance for TNF- $\alpha$ production in non-eosinophilic asthma. A limitation exists when interpreting these findings since asthma medications, such as inhaled corticosteroids, are regularly used to reduce airway inflammation in asthma. Furthermore, airway neutrophils did not respond to LPS stimulation in healthy controls or eosinophilic and non-eosinophilic asthma. Similar findings of unresponsiveness of airway cells to LPS stimulation along with decreased release of TNF- $\alpha$ from airway cells have previously been reported in chronic obstructive pulmonary disease [14], and the mechanisms of this warrant further investigation.

The development of high-throughput screening and genomewide gene expression using microarrays has permitted many diseases to be characterised into groups by gene expression profiling. Analysis of the current data suggests that the type of airway inflammation present can separate asthma into subgroups based on altered systemic neutrophil gene expression profiles. Although relatively small groups were studied here, significant differences in gene expression and distinct dendrograms were observed. In addition, genes in peripheral blood neutrophils from asthma phenotypes with known immune-related functions were identified and confirmed to show altered expression using real-time PCR.

Activation of the innate immune response, including increased expression of the receptors TLR4, TLR2, CD14 and surfactant protein $\mathrm{A}$ and the cytokines IL- 8 and IL-1 $\beta$, has been demonstrated in the airways of participants with noneosinophilic asthma [6]. The present data show altered gene expression profiles and increased resting blood neutrophil IL-8 production in non-eosinophilic asthma. This could both promote the development of non-eosinophilic airway inflammation and influence existing non-eosinophilic airway inflammation. Many (54\%) genes that were differentially expressed in resting neutrophils in non-eosinophilic asthma were also regulated by LPS stimulation, indicating that these genes play a role in neutrophil activation. ABRAHAM et al. [15] demonstrated that there is a significant correlation between peripheral blood neutrophil phenotype and the pulmonary response to endotoxin, i.e. the accumulation of neutrophils and the intensity of the immune response in the airways to endotoxin challenge is directly associated with the activation state of circulating neutrophils.

Large numbers of neutrophils are often present in the airways of participants with non-eosinophilic asthma. Increased accumulation of neutrophils in the airways could be due to enhanced chemotaxis from the blood and/or enhanced survival of these cells. Here, it has been shown that peripheral blood neutrophils show increased expression of genes relating to enhanced cell motility and survival. Genes relating to cell motility that were upregulated in non-eosinophilic asthma include proteins (e.g. IL-8 and S100 calcium-binding protein A8 (S100A8)), receptors (e.g. CC chemokine receptor-like (CCRL) 2) and transcription factors (e.g. serum response factor). These genes are readily expressed in neutrophils and upon neutrophil activation by LPS [16, 17]. Importantly, expression of IL-8 [6], S100A8 [16] and CCRL2 [8] have been associated with neutrophilic lung inflammation.

IL-8 is important for many neutrophil functions, including chemotaxis and survival. Enhanced production of IL-8 by blood neutrophils may prime these cells for their migration to 


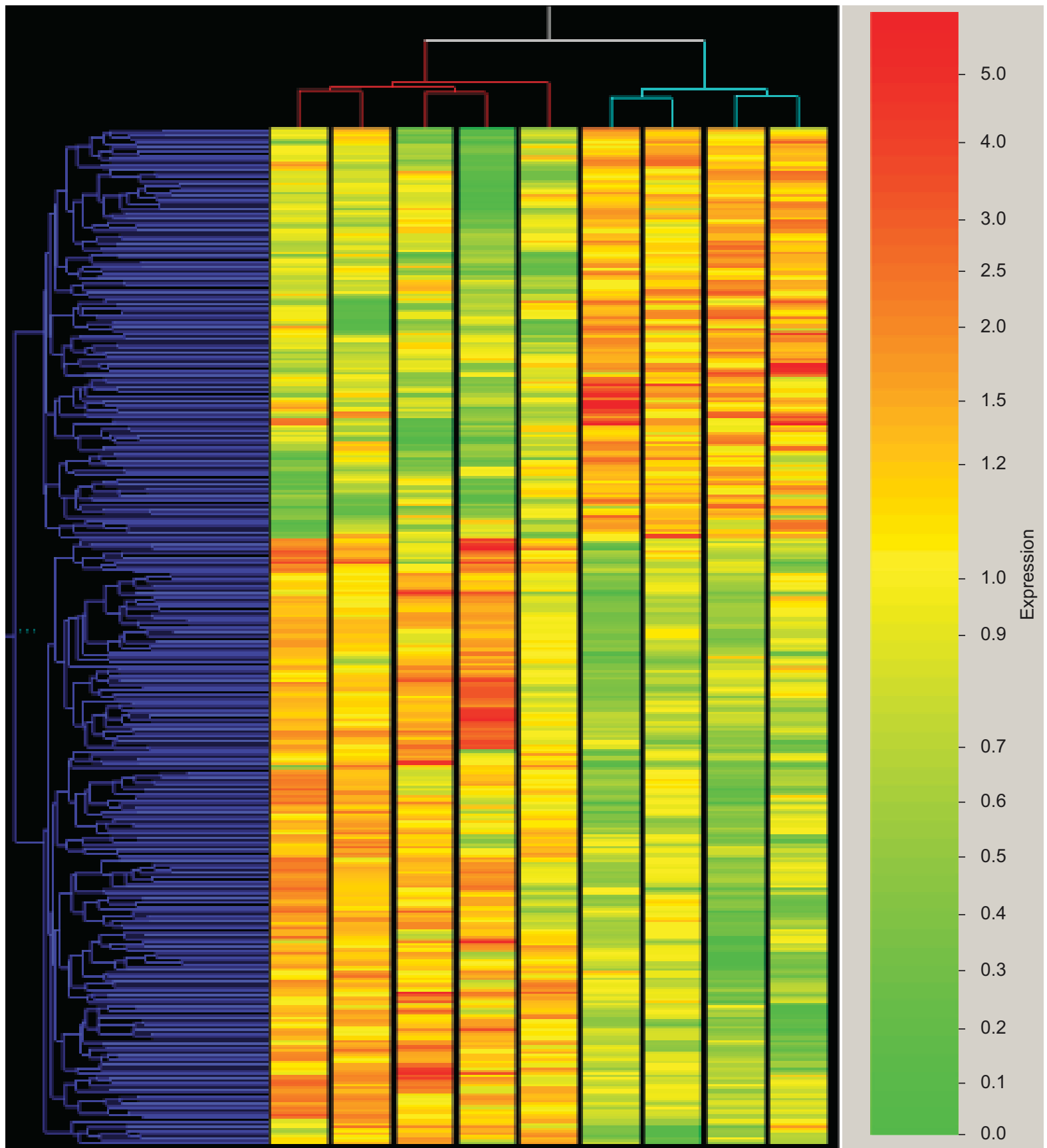

FIGURE 3. Gene expression profiles of resting neutrophils from participants with eosinophilic asthma ( $n=5)$ versus those with non-eosinophilic asthma ( $\mathrm{n}=4)$. The vertical dendrogram represents the relationship between participants with non-eosinophilic (blue branches) and eosinophilic asthma (red branches). The horizontal dendrogram represents the relationship between the expression levels of each gene across all of the samples.

the airways. Circulating levels of IL-8 can also stimulate the bone marrow to release neutrophils into the circulation [18]. Enhanced release of IL-8 has previously been reported in blood neutrophils isolated from patients with cystic fibrosis [19]. Enhanced IL-8 release may be due to positive feedback from the leakage of inflammatory mediators from the airways, release of immature neutrophils from the bone marrow or genetic differences, such as IL-8 gene polymorphisms; however, further investigation is required in order to elucidate this.
There is a considerable amount of literature demonstrating that cell fate is regulated at the level of gene expression [20], and that these changes are important in the resolution of inflammatory processes [21]. Particular examples of genes whose expression was increased in non-eosinophilic asthma and relate to a delay in apoptosis include GADD45B, histone deacetylase (HDAC) 3 and 5, SGK and CEBPB. Several of these genes are thought to increase cell survival through modulation of the NF- $\kappa B$ pathway $[22,23]$. RIPK2 is another important signalling molecule involved in the activation of $N F-\kappa B$ 


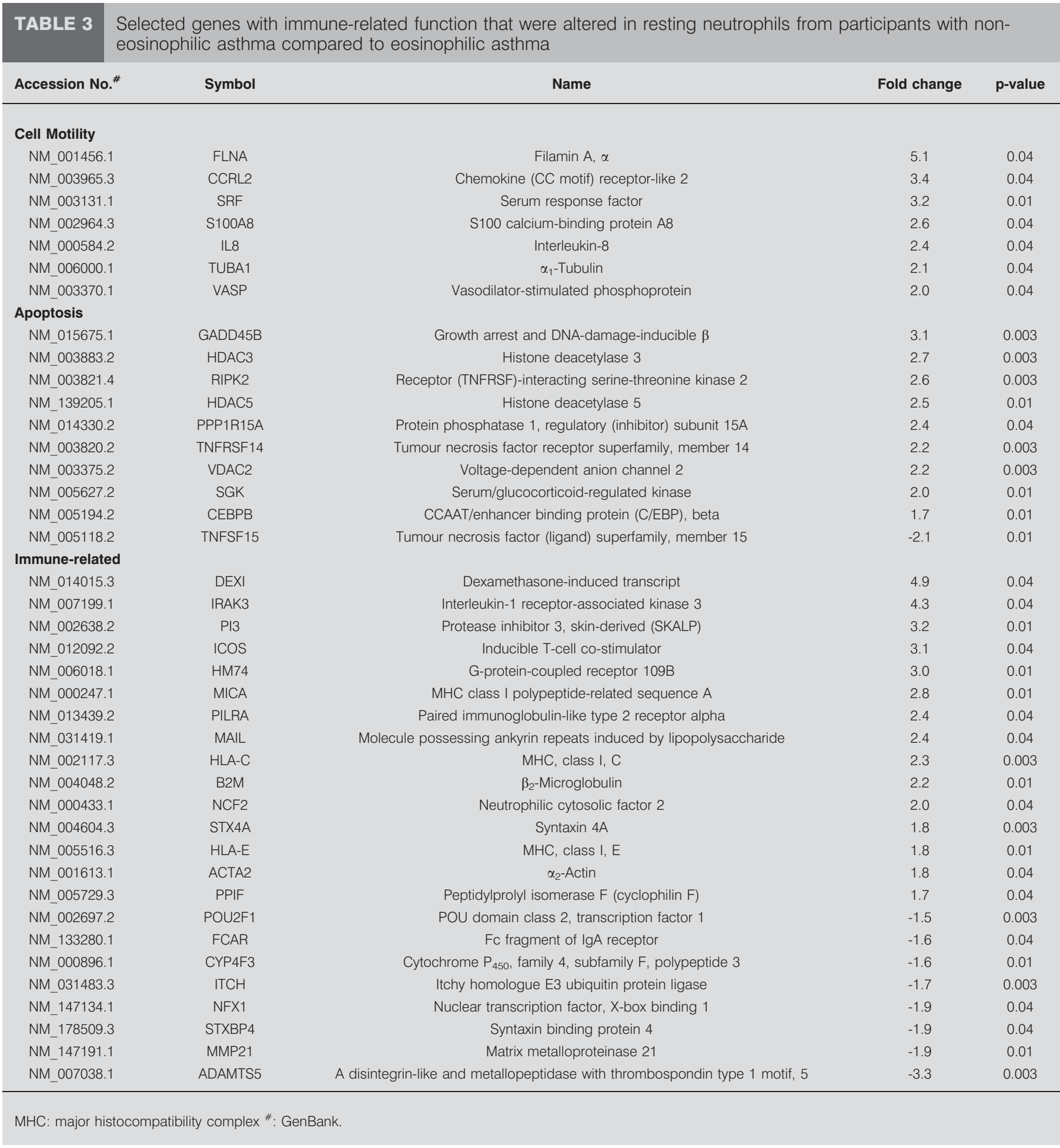

through stimulation of numerous innate immune receptors, including TLR2, TLR4, nucleotide-binding oligomerisation domain proteins, IL-1 receptor (IL-1R) and IL-18R [24]. IRAK-M (IRAK3), a negative regulator of TLR signalling [25] was upregulated in resting neutrophils in non-eosinophilic asthma in the present study, and has recently been linked to the pathogenesis of early-onset persistent asthma [26].
The gene expression changes appear to be clinically relevant since many were correlated with the degree of airway obstruction in asthma, including GADD45B, IRAK3, HM74, MAIL, PI3, syntaxin 4A (STX4A), major histocompatibility complex, class I, E (HLA-E), HDAC5 and TNFRSF14. These genes exhibit a variety of functions, including cell signalling (GADD45B and IRAK3), transcriptional regulation (MAIL and 


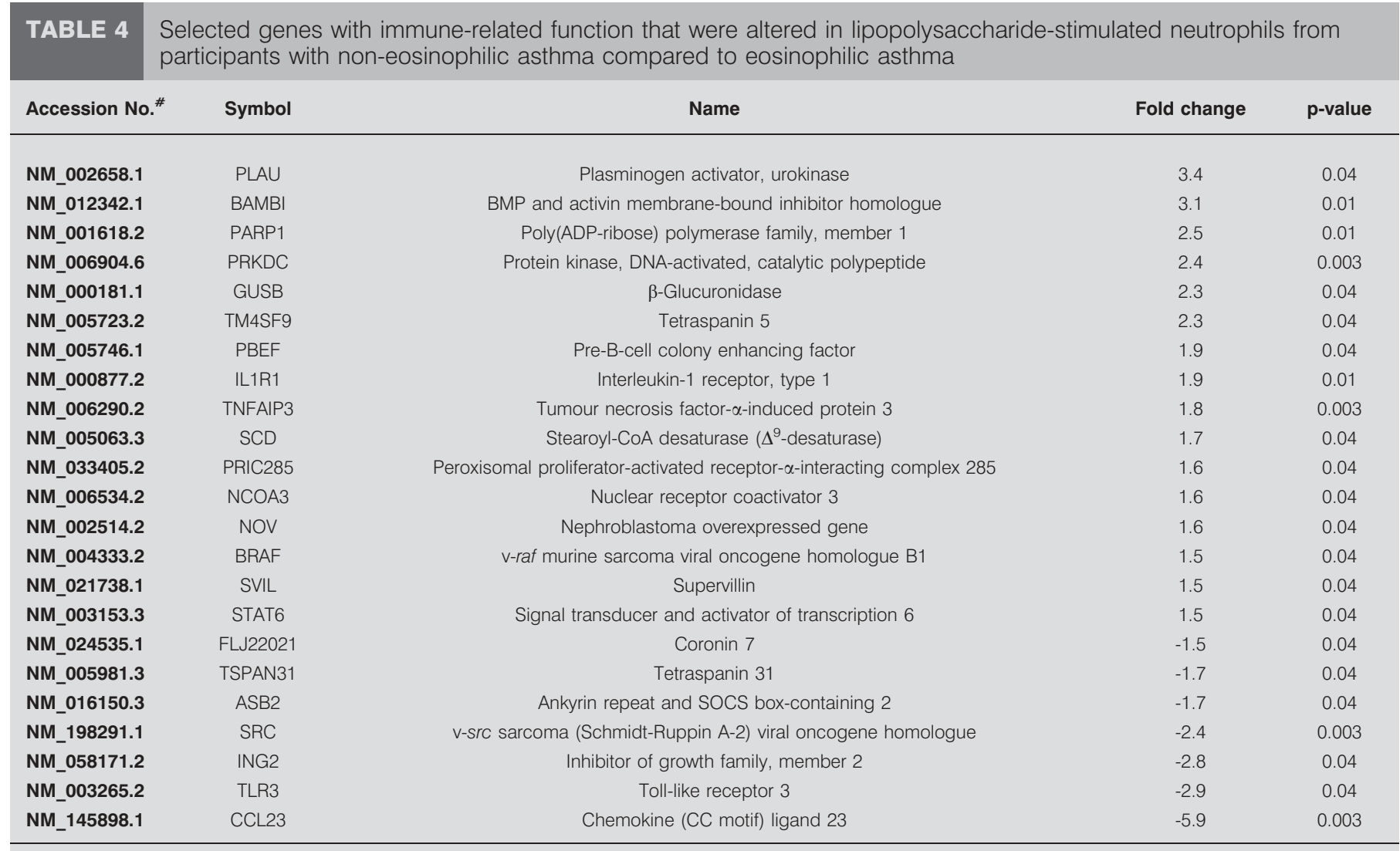

BMP: bone morphogenetic protein; SOCS: suppressor of cytokine signalling. ${ }^{\#}$ : GenBank.

HDAC5), receptor activity (HM74, HLA-E and TNFRSF14), protease inhibition (PI3) and protein transport (STX4A). Importantly, several of these genes participate in the regulation of NF- $\kappa \mathrm{B}$ activity, including GADD45B, IRAK3, MAIL, TNFRSF14 and PI3. This further underscores the importance of this pathway in the mechanisms of non-eosinophilic asthma.

Differences in the response to LPS may also play a role in the innate immune defence against invading microorganisms, and may contribute to airway inflammation. The present study demonstrated significant alterations in gene expression following LPS stimulation in non-eosinophilic asthma compared to eosinophilic asthma. The genes that were altered (e.g. PLAU and IL1R1) suggested that there was a potentiation of LPS responses in non-eosinophilic asthma, and further increases in genes relating to cell survival (e.g. pre-B-cell colony enhancing factor (PBEF); TNF- $\alpha$-induced protein 3 (TNAIF3); v-raf murine sarcoma viral oncogene homologue B1 (BRAF); protein kinase, DNA-activated, catalytic polypeptide (PRKDC) and supervillin (SVIL)).

In addition to this, a decrease in the production of OSM but not OSM mRNA from LPS-stimulated neutrophils was observed in participants with eosinophilic asthma, which was significantly different from that in healthy controls and also lower than in non-eosinophilic asthma. OSM, an IL-6 family cytokine, is thought to promote airway remodelling [27], potentially through increasing the proliferation of both fibroblasts and smooth muscle cells [28] and inducing the production of angiogenic factors, such as vascular endothelial growth factor [29]. Neutrophils have an intracellular store of OSM and produce large concentrations of the protein upon stimulation with inflammatory triggers, such as LPS [30]. Since differences were found in the present study between the production of OSM and its gene expression, future studies should measure the levels of OSM within the intracellular stores of the neutrophil.

These findings provide further evidence that neutrophils are transcriptionally active cells that are responsive to environmental stimuli and capable of a complex series of late transcriptional changes. We have identified specific gene profiles associated with non-eosinophilic and eosinophilic asthma, providing further validation that these phenotypes of asthma involve very different molecular mechanisms of disease pathogenesis at the systemic level. There is altered production of both IL-8 and OSM, indicating differential activation of neutrophils in asthma phenotypes. This study highlights the importance of neutrophils in the pathogenesis of non-eosinophilic asthma.

\section{STATEMENT OF INTEREST}

None declared.

\section{REFERENCES}

1 Wenzel SE. Asthma: defining of the persistent adult phenotypes. Lancet 2006; 368: 804-813. 
2 Simpson JL, Scott RJ, Boyle MJ, et al. Inflammatory subtypes in asthma: assessment and identification using induced sputum. Respirology 2006; 11: 54-61.

3 Douwes J, Gibson P, Pekkanen J, et al. Non-eosinophilic asthma: importance and possible mechanisms. Thorax 2002; 57: 643-648.

4 Berry M, Morgan A, Shaw DE, et al. Pathological features and inhaled corticosteroid response of eosinophilic and non-eosinophilic asthma. Thorax 2007; 62: 1043-1049.

5 Gibson PG, Simpson JL, Saltos N. Heterogeneity of airway inflammation in persistent asthma. Chest 2001; 119: 1329-1336.

6 Simpson JL, Grissell TG, Douwes J, et al. Innate immune activation in neutrophilic asthma and bronchiectasis. Thorax 2006; 62: 211-218.

7 Subrahmanyam YV, Yamaga S, Prashar Y, et al. RNA expression patterns change dramatically in human neutrophils exposed to bacteria. Blood 2001; 97: 2457-2468.

8 Coldren CD, Nick JA, Poch KR, et al. Functional and genomic changes induced by alveolar transmigration in human neutrophils. Am J Physiol Lung Cell Mol Physiol 2006; 291: L1267-L1276.

9 Kobayashi SD, Voyich JM, Braughton KR, et al. Gene expression profiling provides insight into the pathophysiology of chronic granulomatous disease. J Immunol 2004; 172: 636-643.

10 Malcom KC, Arndt PG, Manos EJ, et al. Microarray analysis of lipopolysaccharide-treated human neutrophils. Am J Physiol Lung Cell Mol Physiol 2003; 284: L663-L670.

11 Zhang X, Kluger $Y$, Nakayama $Y$, et al. Gene expression in mature neutrophils: early responses to inflammatory stimuli. J Leukoc Biol 2004; 75: 358-372.

12 Gibson PG, Wlodarczyk JW, Hensley MJ, et al. Epidemiological association of airway inflammation with asthma symptoms and airway hyperresponsiveness in childhood. Am J Respir Crit Care Med 1998; 158: 36-41.

13 Grissell TV, Powell H, Shafren DR, et al. Interleukin-10 gene expression in acute virus-induced asthma. Am J Respir Crit Care Med 2005; 172: 433-439.

14 Dentener MA, Louis R, Cloots RHE, et al. Differences in local versus systemic TNF $\alpha$ production in COPD: inhibitory effect of hyaluran on LPS induced blood cell TNF $\alpha$ release. Thorax 2006; 61: 478-484.

15 Abraham E, Nick JA, Azam T, et al. Peripheral blood neutrophil activation patterns are associated with pulmonary inflammatory responses to lipopolysaccharide in humans. J Immunol 2006; 176: 7753-7760.

16 Bozinovski S, Cross M, Vlahos R, et al. S100A8 chemotactic protein is abundantly increased, but only a minor contributor to
LPS-induced, steroid resistant neutrophilic lung inflammation in vivo. J Proteome Res 2005; 4: 136-145.

17 Martinez FO, Sironi M, Vecchi A, et al. IL-8 induces a specific transcriptional profile in human neutrophils: synergism with LPS for IL-1 production. Eur J Immunol 2004; 34: 2286-2292.

18 van Eeden SF, Terashima T. Interleukin-8 (IL-8) and the release of leukocytes from the bone marrow. Leuk Lymphoma 2000; 37: 259-271.

19 Corvol H, Fitting C, Chadelat K, et al. Distinct cytokine production by lung and blood neutrophils from children with cystic fibrosis. Am J Physiol Lung Cell Mol Physiol 2003; 284: L997-L1003.

20 Kobayashi SD, Voyich JM, Buhl CL, et al. Global changes in gene expression by human polymorphonuclear leukocytes during receptor-mediated phagocytosis: cell fate is regulated at the level of gene expression. Proc Natl Acad Sci USA 2002; 99: 6901-6906.

21 O'Neill AJ, Doyle BT, Molloy E, et al. Gene expression profile of inflammatory neutrophils: alterations in the inhibitors of apoptosis proteins during spontaneous and delayed apoptosis. Shock 2004; 21: 512-518.

22 Zhang L, Cui R, Cheng X, et al. Antiapoptotic effect of serum and glucocorticoid-inducible protein kinase is mediated by novel mechanism activating IкB kinase. Cancer Res 2005; 65: 457-464.

23 De Smaele E, Zazzeroni F, Papa S, et al. Induction of gadd $45 \beta$ by NF- $\mathrm{B}$ downregulates pro-apoptotic JNK signalling. Nature 2001; 414: 308-313.

24 Kobayashi K, Inohara N, Hernandez LD, et al. RICK/Rip2/ CARDIAK mediates signalling for receptors of the innate and adaptive immune systems. Nature 2002; 416: 194-199.

25 Kobayashi K, Hernandez LD, Galan JE, et al. IRAK-M is a negative regulator of Toll-like receptor signaling. Cell 2002; 110: 191-202.

26 Balaci L, Spada MC, Olla N, et al. IRAK-M is involved in the pathogenesis of early-onset persistent asthma. Am J Hum Genet 2007; 80: 1103-1114.

27 James AL, Wenzel SE. Clinical relevance of airway remodelling in airway diseases. Eur Respir J 2007; 30: 134-155.

28 Grove RI, Eberhardt C, Abid S, et al. Oncostatin M is a mitogen for rabbit vascular smooth muscle cells. Proc Natl Acad Sci USA 1993; 90: 823-827.

29 Faffe DS, Flynt L, Mellema L, et al. Oncostatin M causes VEGF release from human airway smooth muscle: synergy with IL-1 $\beta$. Am J Physiol Lung Cell Mol Physiol 2005; 288: L1040-L1048.

30 Grenier A, Dehoux M, Boutten A, et al. Oncostatin M production and regulation by human polymorphonuclear neutrophils. Blood 1999; 93: 1413-1421. 